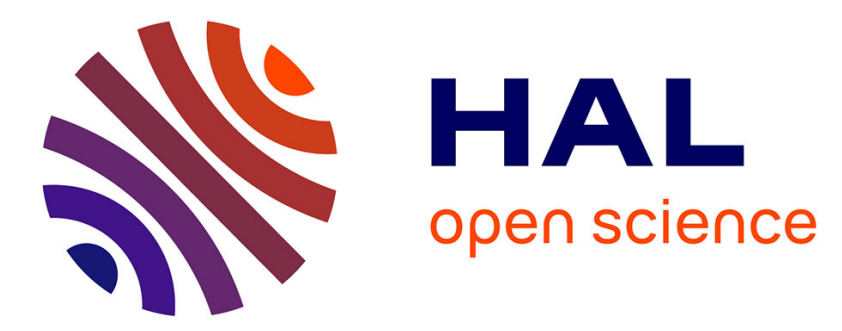

\title{
Failure diagnosis and nonlinear observer. Application to a hydraulic process
}

\author{
Hassan Hammouri, Pousga Kabore, Sami Othman, Jean Biston
}

\section{To cite this version:}

Hassan Hammouri, Pousga Kabore, Sami Othman, Jean Biston. Failure diagnosis and nonlinear observer. Application to a hydraulic process. Journal of the Franklin Institute of the State of Pennsylvania, 2002, 339 (4-5), pp.455-478. 10.1016/S0016-0032(02)00027-3 . hal-00524444

\section{HAL Id: hal-00524444 \\ https://hal.science/hal-00524444}

Submitted on 11 Oct 2010

HAL is a multi-disciplinary open access archive for the deposit and dissemination of scientific research documents, whether they are published or not. The documents may come from teaching and research institutions in France or abroad, or from public or private research centers.
L'archive ouverte pluridisciplinaire HAL, est destinée au dépôt et à la diffusion de documents scientifiques de niveau recherche, publiés ou non, émanant des établissements d'enseignement et de recherche français ou étrangers, des laboratoires publics ou privés. 
This document must be cited according to its final version which is published in a journal as:

H. Hammouri ${ }^{1}$, P.Kabore ${ }^{1}$, S. Othman ${ }^{1}$, J. Biston ${ }^{1}$

"Failure diagnosis and nonlinear observer.

Application to a hydraulic process",

Journal of the Franklin Institute of the State of Pennsylvania

339, 4-5 (2002) 455-478

This final version may be found:

http://dx.doi.org/10.1016/S0016-0032(02)00027-3

All open archive documents of $\mathbf{H}$. Hammouri are available at: http://hal.archives-ouvertes.fr/HAMMOURI-HASSAN

All open archive documents of $\boldsymbol{H}$. Hammouri research group (SNLEP) are available at:

http://hal.archives-ouvertes.fr/SNELP

http://www.tinyurl.com/SNELP

The professional web page (Fr/En) of $\mathrm{H}$. Hammouri is:

http://www.lagep.univ-lyon1.fr/signatures/hammouri.hassan

1

Université de Lyon, Lyon, F-69003, France; Université Lyon 1;

CNRS UMR 5007 LAGEP (Laboratoire d'Automatique et de GEnie des Procédés),

43 bd du 11 novembre, 69100 Villeurbanne, France

Tel +33 (0) 472431845 - Fax +33 (0) 472431699

http://www-lagep.univ-lyon1.fr/ http://www.univ-lyon1.fr http://www.cnrs.fr 


\title{
Failure Diagnosis and Nonlinear Observer. Application to a Hydraulic Process. \\ H. Hammouri, P. Kabore, S. Othman and J. Biston \\ LAGEP, UPRES-A CNRS Q 5007/ CPE Bat G308, Universite Claude \\ Bernard, 43 bd du 11 Nov. 1918, 69622 Villeurbanne Cedex \\ e-mail: hammouri@lagep.cpe.fr
}

\begin{abstract}
This paper deals with the problem of fault detection and isolation for nonlinear systems. The proposed approach is based on nonlinear disturbance decoupling techniques and nonlinear observers. First, we give sufficient conditions and a design procedure for the synthesis of residual generators. Next, we characterize a class of nonlinear systems for which a high gain observer can be designed. Finally, the two above developments are illustrated through a real application dealing with the detection and isolation of three failures in a hydraulic process. The design of the residual generators is shown and their performances are discussed.
\end{abstract}

Key words: Nonlinear system, Diagnosis, Observer, Hydraulic process.

\section{Introduction}

Over the last two decades, fault diagnosis has become an issue of primary importance in modern process automation as it provides a basis for the reliable and safe fundamental design features of many complex engineering systems. Failure diagnosis consists of providing information on the time and on the location of faults that occur in the supervised process. The former task is called fault detection problem while the latter is referred to as fault isolation. Several methods for Fault Detection and Isolation (FDI) have been presented since the original works of Beard [3] and Jones [23]. The most important ones are based on the concept of analytical redundancy, which consists of observing certain variables that are identically equal to zero (or under a predetermined threshold) as long as no failure occurs. Such observed signals are called residuals, and their generating system is referred to as a residual generator. If a residual is not altered by a subset of failures, we say that this residual is unaffected by the subset. In fact, such a residual is aptly known as a structured residual. Making a diagnosis therefore consists of comparing various structured residuals with different decoupling properties. The analysis of the distinguishably nonzero residuals leads to fault isolation. Parity space techniques (see e.g. [13] and the references therein), parameter estimation techniques (see e.g. [21], [7], [9] ), statistical techniques [2] and observer-based techniques (see e.g. [26]; [27]; [8]; [29]; [1]; [18]; [19] and the references therein) are amongst the most important contributions to the analytical redundancy-based failure diagnosis approach.

Many solutions for the FDI problem that use an observer-based approach have been proposed. These include an Eigenstructure Assignment approach, an Unknown Input Observer approach , and a geometric approach proposed in [26] and [27] (see e.g. [8]; [29] as surveys of existing techniques). In fact, observers are used to estimate a part of the state vector which allows the reconstruction of 
the output functions and the innovation is then taken as a residual. The choice of such output functions depends on their decoupling properties with respect to failures ([26]; [27]). For nonlinear systems, the FDI problem was initially introduced by P. M. Frank [7]. More recently, many technics were proposed in order to solve the FDI problem for nonlinear systems:

- L. R. Seliger and P. M. Frank [31] propose some solutions based on robust observer approach.

- Combining the geometric decoupling technics together with the nonlinear observer synthesis, the authors in ([30], [1]; [18]; [19]; [20]) gave sufficient conditions permetting to solve the FDI problem.

The outline of this paper is as follows. In section 2, we define a sufficient condition for the solution of the FDI problem for of nonlinear systems. In section 3 , we show how one can extend the high gain observer (stated in [11]) to a more general class of nonlinear systems. In section 4, we apply the theoretical results stated in sections 2 and 3 to a hydraulic process.

\section{Fault detection and isolation for nonlinear systems}

We consider the following class of nonlinear systems:

$$
\left\{\begin{array}{l}
\dot{x}=f(x)+\sum_{i=1}^{m} u_{i} g_{i}(x)+\sum_{i=1}^{m_{f}} v_{i} e_{i}(x) \\
y=h(x)
\end{array}\right.
$$

where the state $x(t) \in V$ is an open set of $\mathbb{R}^{n}$, the input $u(t)=\left(u_{1}(t), \ldots, u_{m}(t)\right) \in$ $U$ is an open set of $\mathbb{R}^{m}$ and the output $y(t)=\left(y_{1}(t), \ldots, y_{p}(t)\right) \in \mathbb{R}^{p}$, the $v_{i}$ are unknown scalar failure modes and the vector failure $\underline{v}_{i}$ is given by $\left(v_{1}, \ldots, v_{i-1}, v_{i+1}, \ldots, v_{m_{f}}\right)$.

The following definition extends the definition of the FPRG (fundamental problem in residual generation) proposed by M. A. Massoumnia et al. in [27].

Definition 1 The FDI w.r.t. $v_{i}$ admits a solution if we can find a dynamical system called residual filter (or residual generator):

$$
\begin{aligned}
\dot{z} & =F(u, z, y) \\
r & =H(y, z)
\end{aligned}
$$

satisfying the following conditions:

C1) The residual $r$ depends upon $v_{i}$ in the following sense: There exists an initial state $(x(0), z(0))$ of the augmented system (1), (2) and time varying signals $u, \underline{v}_{i}$ such that the input-output map $v_{i} \rightarrow r\left(x(0), z(0), u, \underline{v}_{i}, v_{i}\right)$ is not trivial one (It is not a constant map).

C2) If $v_{i}=0$, then

i) For every initial state $(x(0), z(0))$ of (1), (2) and for every signals $u, \underline{v}_{i}$, $\lim _{t \rightarrow \infty} r(t)=0$

ii) $r$ doesn't depend on $\underline{v}_{i}$. 
For linear systems, the residual generator proposed by Massoumnia et al. is a linear one, and the authors gave a geometric condition (necessary and sufficient condition) that allows them to solve the FDI problem of definition 1.

In what follows, we give a sufficient condition together with a design procedure to solve the FDI w.r.t. $v_{1}$. Concerning the standard geometric notions of distributions, the reader is referred to the well-known books of Isidori [22], and Nijmeijer and Van Der Schaft [28].

For the sake of simplicity, we will consider the FDI w.r.t. $v_{1}(i=1)$. Consider the following distributions $\Delta_{1} \subset \Delta_{2} \subset \ldots \subset \Delta_{k-1} \subset \Delta_{k} \subset \ldots$ where: $\Delta_{1}$ is spanned by $\left\{e_{2}, \ldots, e_{m_{f}}\right\}$ and for $k \geq 2, \Delta_{k}$ is the distribution spanned by $\Delta_{k-1}$ and $\left[\mathcal{G}, \Delta_{k-1}\right]$ where $\mathcal{G}=\left\{f, g_{1}, \ldots, g_{m}\right\}$ and $\left[\mathcal{G}, \Delta_{k-1}\right]$ denotes the family of all vector fields $[X, Y], X \in \mathcal{G}$ and $Y \in \Delta_{k-1}$, [,] is the Lie Bracket operator.

Assuming that the $\Delta_{i}$ 's are of constant dimension (i.e. $\operatorname{dim} \Delta_{i}(x)=\operatorname{dim}$ $\left.\Delta_{i}\left(x^{\prime}\right), \forall x, x^{\prime} \in V, i=1,2, \ldots\right)$. Then, there exists an integer $k$ such that:

$$
\forall i \geq k, \Delta_{i+1}=\Delta_{i}=\Delta^{*}
$$

Now, consider the functional space $\Omega$ of smooth functions $\sigma: \mathbb{R}^{p} \longrightarrow \mathbb{R}$ such that $L_{X}\left(\sigma \circ h_{j}\right)=0$, for $j=1, . ., p$. Here $L_{X}(\psi)=\sum_{l=1}^{m} X_{i} \frac{\partial \psi}{\partial x_{i}}$ is the Lie derivative of $\psi$ w.r.t. the vector field $X$. Noticing that $\Omega$ is not empty (since 0 is a solution). Denote by $d \Omega$ the co-distribution spanned by all one-forms $d\left(\sigma \circ h_{j}\right), \sigma \in \Omega$ and assume that $d \Omega$ is of constant dimension on $V$. Let $\varphi_{1}, \ldots, \varphi_{\bar{p}} \in \Omega$ such that $\left(d \varphi_{1}, \ldots, d \varphi_{\bar{p}}\right)$ forms a basis $d \Omega$. In what follows, $\varphi$ denotes the vector map $\left[\begin{array}{l}\varphi_{1} \\ \vdots \\ \varphi_{\bar{p}}\end{array}\right]$.

Now, consider the nominal system (1) in which $v_{2}=\ldots=v_{m_{f}}=0$ with the output map $\varphi \circ h$ :

$$
\left\{\begin{array}{l}
\dot{x}=f(x)+\sum_{i=1}^{m} u_{i} g_{i}(x) \\
\bar{y}=\varphi \circ h(x)=\bar{h}(x)=\left(\bar{h}_{1}(x), \ldots, \bar{h}_{\bar{p}}(x)\right)^{\top}
\end{array}\right.
$$

Denote by $O_{\varphi \circ h}$ the observation space of system (3): the smallest real vector space containing $\bar{h}_{1}, \ldots, \bar{h}_{\bar{p}}$ and such that for every $\sigma \in O_{\varphi \circ h}, L_{X} \sigma \in O_{\varphi \circ h}$, where $X=f+\sum_{i=1}^{m} u_{i} g_{i},\left(u_{1}, \ldots, u_{m}\right)$ is any m-tuple of $U$. Assume that the codistribution defined by $d O_{\varphi \circ h}$ is of constant dimension on $V$ and $\left(d \Psi_{1}, \ldots, d \Psi_{d}\right)$ is a basis of $d O_{\varphi \circ h}$. Consider a local system of coordinates $\xi=\left(\xi_{1}, \ldots, \xi_{n}\right)$ such that $\xi_{i}=\Psi_{i}(x)$ for $i=1, \ldots, d$, and set $\xi^{1}=\left(\xi_{1}, \ldots, \xi_{d}\right)^{\top}, \xi^{2}=\left(\xi_{d+1}, \ldots, \xi_{n}\right)^{\top}$, we obtain: 
Lemma 2 In this system of coordinates, system (3) takes the following form:

$$
\begin{aligned}
\dot{\xi}^{1} & =F^{1}\left(\xi^{1}\right)+\sum_{i=1}^{m} u_{i} G_{i}^{1}\left(\xi^{1}\right) \\
\dot{\xi}^{2} & =F^{2}(\xi)+\sum_{i=1}^{m} u_{i} G_{i}^{2}(\xi) \\
\bar{y} & =H\left(\xi^{1}\right)
\end{aligned}
$$

The decomposition (4), (5), (6) is well-known and can be found in the general literature on nonlinear systems. In what follows, we assume that the system of coordinates $\left(\xi_{1}, \ldots, \xi_{n}\right)$ is a global one: i.e. the map $x \mapsto\left(\xi_{1}(x), \ldots, \xi_{n}(x)\right)$ is a diffeomorphism from $V$ into its range. If this is not the case, we restrict ourselves to an open subset of $V$ on which $\left(\xi_{1}, \ldots, \xi_{n}\right)$ is well defined.

Now, denote by ker $d O_{\varphi \circ h}$ the distribution spanned by vector fields $X$ such that $L_{X}(\psi)=0$ for every $\psi \in O_{\varphi \circ h}$.

We can state our main result :

Theorem 3 Assume that the following conditions hold:

i) $e_{1} \notin \operatorname{ker} d O_{\varphi \circ h}$

ii) system (4), (6) admits an asymptotic observer then the FDI w.r.t. $v_{1}$ possesses a solution.

Proof. In what follows, we will construct a residual filter that can be used to detect and isolate the failure mode $v_{1}$ (in other words, a residual generator satisfying conditions $\mathrm{C} 1$ ) and $\mathrm{C} 2$ ) of definition 1 ). To do so, using the construction of $\Delta^{*}$ and the definition of $\varphi \circ h$, we obtain $L_{e_{j}}(\tau)=0$ for $2 \leq j \leq m_{f}$ and for every $\tau \in O_{\varphi \circ h}$. In particular, $L_{e_{2}}\left(\xi_{i}\right)=\ldots=L_{e_{m_{f}}}\left(\xi_{i}\right)=0$, for $1 \leq i \leq d$. form:

Using lemma 2 and taking into account this last fact, system (1) takes the

$$
\begin{gathered}
\dot{\xi}^{1}=F^{1}\left(\xi^{1}\right)+\sum_{i=1}^{m} u_{i} G_{i}^{1}\left(\xi^{1}\right)+v_{1} E_{1}^{1}(\xi) \\
\dot{\xi}^{2}=F^{2}(\xi)+\sum_{i=1}^{m} u_{i} G_{i}^{2}(\xi)+\sum_{i=1}^{m_{f}} v_{i} E_{i}^{2}(\xi) \\
\bar{y}=H\left(\xi^{1}\right)
\end{gathered}
$$

From condition $i i)$, system (4), (6) admits an observer which takes the general form:

$$
\left\{\begin{array}{l}
\dot{\zeta}=\Lambda\left(\zeta, u, \bar{y}-H\left(\hat{\xi}^{1}\right)\right) \\
\hat{\xi}^{1}=\Phi(\zeta)
\end{array}\right.
$$
$\xi^{1}$.

where $\bar{y}$ is the output of system (4), (6) and $\hat{\xi}^{1}$ is the variable which estimates 
Our candidate residual generator is then of the form:

$$
\begin{aligned}
\dot{\zeta} & =\Lambda(\zeta, u, \bar{y}-H(\Phi(\zeta))) \\
r & =H(\Phi(\zeta))-\bar{y}
\end{aligned}
$$

where $\bar{y}$ is the output of (7), (8). In what follow, we will show that system (10) satisfies conditions $C 1$ ), $C 2$ ) of definition 1.

Condition $\mathrm{C} 2)$ :

Condition $C 2)-i$ ), comes from the fact that system (10) is an asymptotic observer for system (7), (9) in which $v_{1}=0$.

Condition C2)-ii). Using the structure of system (7), (9), it is easy to see that if $v_{1}=0$, then $\bar{y}$ doesn't depend on $v_{2}, \ldots, v_{m_{f}}$ and in similar way, r doesn't depend on $v_{2}, \ldots, v_{m_{f}}$.

Condition $\mathrm{C} 1$ ):

There exists an initial state $(x(0), z(0))$ of the augmented system (1), (2) and time varying signals $u, \underline{v}_{i}$ such that the Frechet derivative of the functional map $v_{i} \rightarrow r\left(x(0), z(0), u, \underline{v}_{i}, v_{i}\right)$ does not vanish.

Assume that $C 1)$ is not satisfied. Then for every initial state $(\xi(0), \varsigma(0))$ of the augmented system (7), (8) and (10) and every signals $u,\left(v_{2}, \ldots, v_{m_{f}}\right)=\underline{v}_{i}$, the Frechet derivative of the functional map $v_{i} \rightarrow r\left(x(0), z(0), u, \underline{v}_{i}, v_{i}\right)$ is equal to zero. This means that the residual $r$ doesn't depend on $v_{1}$. using the structure of system (10), it follows that $\bar{y}$ doesn't depend on $v_{1}$. This implies that the output $\bar{y}$ of system (7), (8), (9) in which $v_{2}=\ldots=v_{m_{f}}=0$ doesn't depend on $v_{1}$. Set $E_{1}(\xi)=\left[E_{1}^{1 T}(\xi), E_{1}^{2 T}(\xi)\right]^{T}$ and applying proposition 4.14 (cf pp 136-137 [28]), it follows that for every $\tau \in O_{H}, L_{E_{1}}(\tau)=0$ (where $O_{H}$ is the observability space of system (7), (8), (9)), or equivalently, $e_{1} \in \operatorname{ker} d O_{\varphi \circ h}$. This contradicts condition i) of theorem 3 . Consequently, $C 1$ is true.

To end this section, we will extend this result to more general systems.

Consider the following control affine system:

$$
\begin{aligned}
\dot{x} & =f(x)+\sum_{i=1}^{m} u_{i} g_{i}(x)+\tilde{f}(x)+\sum_{i=1}^{m} u_{i} \tilde{g}_{i}(x)+\sum_{i=1}^{m} v_{i} e_{i}(x) \\
y & =h(x)
\end{aligned}
$$

Using the above procedure and consider $\Delta^{*}$ and $O_{\varphi \circ h}$ obtained from $\left\{f, g_{1}, \ldots, g_{m}\right\}$ and $\left\{e_{2}, \ldots, e_{m f}\right\}$. Let $\left(d \Psi_{1}, \ldots, d \Psi_{d}\right)$ be a basis of $d O_{\varphi \circ h},\left(\xi_{1}, \ldots, \xi_{n}\right)$ be a system of coordinates s.t. $\left(\xi_{1}, \ldots, \xi_{d}\right)=\left(\Psi_{1}(x), \ldots, \Psi_{d}(x)\right)$ and assume the following hypothesis:

$H 1)$ For $1 \leq i \leq d, \quad L_{\tilde{f}}\left(\psi_{i}\right), L_{\tilde{g}_{j}}\left(\psi_{i}\right)$ depend only on $\left(\xi_{1}, \ldots, \xi_{d}\right)$ and $y=h(x)$. It means that:

$$
\begin{aligned}
L_{\tilde{f}}\left(\xi_{i}\right) & =\tilde{F}_{i}\left(\xi^{1}, y\right), L_{\tilde{g}_{j}}\left(\xi_{i}\right)=\tilde{G}_{i j}\left(\xi^{1}, y\right) \\
\text { for } 1 & \leq i \leq d
\end{aligned}
$$

As above (see lemma 2), system (11) with output $\bar{y}=\varphi \circ h$ takes the following 
form:

$$
\begin{aligned}
\dot{\xi}^{1} & =F^{1}\left(\xi^{1}\right)+\sum_{i=1}^{m} u_{i} G_{i}^{1}\left(\xi^{1}\right)+\tilde{F}^{1}\left(\xi^{1}, y\right)+\sum_{i=1}^{m} u_{i} \tilde{G}_{i}^{1}\left(\xi^{1}, y\right)+v_{1} E_{1}^{1}(\xi)(1 \\
\dot{\xi}^{2} & =F^{2}(\xi)+\sum_{i=1}^{m} u_{i} G_{i}^{2}(\xi)+\tilde{F}^{2}(\xi)+\sum_{i=1}^{m} u_{i} \tilde{G}_{i}^{2}(\xi)+\sum_{i=1}^{m_{f}} v_{i} E_{i}^{2}(\xi) \\
\bar{y} & =H\left(\xi^{1}\right)
\end{aligned}
$$

Now, we can state the following extension of theorem 3

Theorem 4 Under hypothesis H1), the FDI w.r.t. $v_{1}$ has a solution if the following conditions hold:

i) $e_{1} \notin \operatorname{ker} d O_{\varphi \circ h}$

ii) For $v_{1}=0$, system (13), (15) admits an asymptotic observer

The proof of this theorem is similar to that of theorem 3 .

\section{Observer Synthesis}

In this section, we give some observer algorithms that can be used in the construction of residual filters. Several contributions to nonlinear observer synthesis can be found in the literature:

- A Luenberger observer can be extended to a linear system up to output injection. Nonlinear systems which can be steered by a change of coordinates to such systems are characterized in [24], [25] and [32].

- The Kalman observer approach has been extended to state affine systems up to output injection by the authors in [5] and [14]. The characterization of nonlinear systems which are equivalent (using a change of coordinates) to such systems has been stated in [15], [16] and [17].

- The High gain observer is a general nonlinear extension of a Luenberger observer. Systems which are concerned by this observer design, are those which are uniformly observable. A nonlinear system is called uniformly observable if for every two different initial states $x, \bar{x}$ and every admissible control, the associated outputs $y(x, u, t), y(\bar{x}, u, t)$ are not identically equal on their time interval of definition. In [10] (see for a short proof [11]), the authors showed for the single output case that if a nonlinear system of the form (1) (in the absence of faults) is uniformly observable, then locally generically the following map:

$$
\left(h, L_{f}(h), \ldots, L_{f}^{n-1}(h)\right)^{T}
$$


is a local diffeomorphism which transforms system (1) into the following form:

$$
\left\{\begin{array}{l}
\dot{x}(t)=A x(t)+\gamma(u(t), x(t)) \\
y(t)=C x(t)
\end{array}\right.
$$

where $\gamma_{i}(x, u)=\gamma_{i}\left(x_{1}, \ldots, x_{i}, u\right)$,

$$
A=\left(\begin{array}{cccc}
0 & 1 & \ldots & 0 \\
\vdots & \ddots & \ddots & \vdots \\
0 & 0 & \ddots & 1 \\
0 & 0 & \ldots & 0
\end{array}\right) \text { and } C=\left[\begin{array}{lll}
1 & 0 \ldots
\end{array}\right]
$$

Moreover, the authors in [11], showed that an observer for (17) takes the following form:

$$
\dot{\hat{x}}=A \hat{x}+\gamma(u, \hat{x})-S_{\theta}^{-1} C^{\top}(C \hat{x}-y)
$$

where $S_{\theta}$ is the symmetric positive definite matrix satisfying:

$$
\theta S_{\theta}+A^{\top} S_{\theta}+S_{\theta} A=C^{\top} C
$$

Several extensions of this observer can be found in [4], [6], [12], ...

In what follows, we will extend the above observer synthesis to a more general class of nonlinear system.

\subsection{Single output case}

Consider the class of nonlinear system of the form:

$$
\left\{\begin{array}{l}
\dot{x}(t)=a(s(t), u(t)) A x(t)+\gamma(s(t), u(t), x(t)) \\
y(t)=C x(t)
\end{array}\right.
$$

where $A, C$ are as above, $s(t), u(t)$ are known signal and input, and $\gamma$ possesses the triangular structure: $\gamma_{i}(s, u, x)=\gamma_{i}\left(s, u, x_{1}, \ldots, x_{i}\right)$.

To design an observer for system (18), we will need the following hypotheses:

H2) We assume that one of the following conditions hold:

i) $0<\alpha<a(s(t), u(t))<\beta$

ii) $0>-\alpha>a(s(t), u(t))>-\beta$

for every $t \geq 0$, where $\alpha$ and $\beta$ are constants.

H3) $\gamma(s, u, x)$ is a global Lipschitz function w.r.t. $x$ locally uniformly w.r.t. $(s, u): \exists M_{1}, M_{2}>0 ; \forall s, u$ with $\|s\| \leq M_{1},\|u\| \leq M_{2} ; \exists \gamma_{0}>0 ; \forall x, \bar{x}$, we have $\|\gamma(s, u, x)-\gamma(s, u, \bar{x})\| \leq \gamma_{0}\|x-\bar{x}\|$. 
Remark 5 a) If condition H2) - ii) holds, then after a simple change of coordinates, the obtained system takes the form (18) and satisfies condition $H 2)-i$ ) (the change of coordinates can take the form $z=\delta x$, where $\delta$ is the $n \times n$ diagonal matrix $\operatorname{diag}\left(\delta_{1}, \ldots, \delta_{i}\right)$ and $\left.\delta_{i}=(-1)^{i}\right)$.

b) Hypothesis H3) can be omitted in the case when trajectories of system (17) are bounded. Indeed, in this case, we can prolong the nonlinear term of system (18) to a global Lipschitz function (see for instance [11]).

Theorem 6 Assume that hypotheses H2), H3) hold, then for every constant $\rho \geq \frac{\beta}{2}$, the following system:

$$
\dot{\hat{x}}=a(s, u) A \hat{x}+\gamma(s, u, \hat{x})-\rho S_{\theta}^{-1} C^{\top}(C \hat{x}-y)
$$

is an exponential observer, for $\theta$ sufficiently large where $S_{\theta}$ is as above.

Proof. We proceed as in [11]:

Set $e(t)=\hat{x}(t)-x(t)$, and consider the change of coordinates: $\varepsilon=\Delta_{\theta} e$, where $\Delta_{\theta}=\operatorname{diag}\left(\frac{1}{\theta}, . ., \frac{1}{\theta^{n}}\right)$ we get:

$$
\dot{\varepsilon}=\theta\left[a(s(t), u(t)) A-\rho S_{1}^{-1} C^{T} C\right] \varepsilon+\Delta_{\theta} \tilde{\gamma}
$$

where $\tilde{\gamma}=\gamma(s(t), u(t), \hat{x}(t))-\gamma(s(t), u(t), x(t))$ and $S_{1}=S_{\theta}$ for $\theta=1$.

Now set $V(t)=\varepsilon(t)^{T} S_{1} \varepsilon(t)$, a simple calculation gives:

$$
\begin{aligned}
\dot{V} & =-\theta a(s(t), u(t)) V-\theta(2 \rho-a(s(t), u(t)))(C \varepsilon)^{2}+2 \varepsilon^{T} S_{1} \Delta_{\theta} \tilde{\gamma} \\
& \leq-\theta a(s(t), u(t)) V-\theta(2 \rho-a(s(t), u(t)))(C \varepsilon)^{2}+2 \sqrt{V} \sqrt{\left(\Delta_{\theta} \tilde{\gamma}\right)^{T} S_{1}\left(\Delta_{\theta} \tilde{\gamma}\right)}
\end{aligned}
$$

Using the triangular structure of $\tilde{\gamma}$ and hypothesis H3), it follows that:

$$
\sqrt{\left(\Delta_{\theta} \tilde{\gamma}\right)^{T} S_{1}\left(\Delta_{\theta} \tilde{\gamma}\right)} \leq \varkappa\|\varepsilon\|
$$

for $\theta$ sufficiently large, where $\varkappa$ is a constant which does not depend on $\theta$. Thus,

$$
\dot{V} \leq-\theta a(s(t), u(t)) V-\theta(2 \rho-a(s(t), u(t)))(C \varepsilon)^{2}+2 \frac{\varkappa}{\lambda_{\min }} V
$$

where $\lambda_{\min }$ is the smallest eigenvalue of $S_{1}$.

Hence for $\rho \geq \frac{\beta}{2}$, we obtain:

$$
\dot{V} \leq-\left(\theta \alpha-2 \frac{\varkappa}{\lambda_{\min }}\right) V
$$

Finally choose $\theta>2 \frac{\varkappa}{\lambda_{\min }},\|\varepsilon(t)\|$ converges exponentially to zero. 


\subsection{A multi-output case}

In what follows, we will extend the above observer synthesis to the multi-output cascade system:

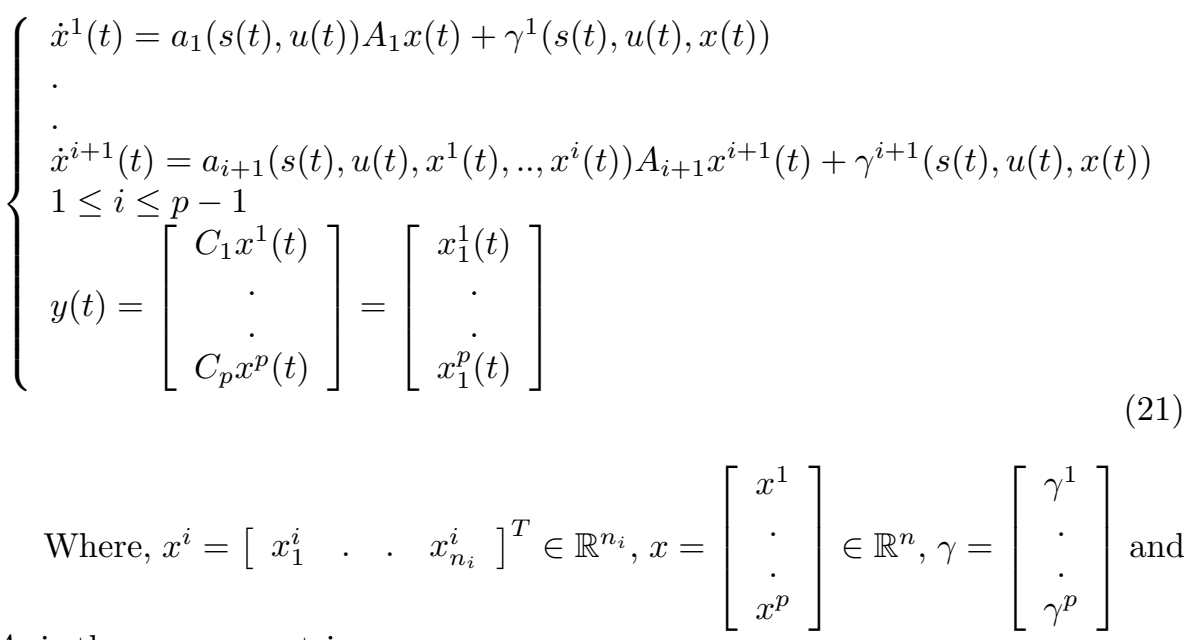
$A_{i}$ is the $n_{i} \times n_{i}$ matrix:

$$
\left(\begin{array}{cccc}
0 & 1 & \ldots & 0 \\
\vdots & \ddots & \ddots & \vdots \\
0 & 0 & \ddots & 1 \\
0 & 0 & \ldots & 0
\end{array}\right)
$$

$s(t), u(t)$ are respectively a known signal and input, and the $a_{i}\left(s, u, x^{1}, . ., x^{i}\right)$ are smooth w.r.t. $\left(x^{1}, . ., x^{i}\right)$.

As above, we assume that the system possesses the following triangular structure:

The $j$ th components of $\gamma^{i}$ satisfy: $\gamma_{j}^{i}(s, u, x)=\gamma_{j}^{i}\left(s, u, . ., x^{i-1}, x_{1}^{i}, . ., x_{j}^{i}\right)$, for $1 \leq j \leq n_{i}$.

To design a high gain observer, we need the following hypotheses:

$H_{4}$ ) We only concerned by a set of bounded admissible controls $\mathcal{U}$ and compact sets $\mathcal{K} \subset \mathcal{K}^{\prime}$ such that for every input $u \in \mathcal{U}$ and every initial state $x \in \mathcal{K}$, the associated trajectory $x_{u}(t)$ belongs to $\mathcal{K}^{\prime}$ for every $t \geq 0$.

$H_{5}$ ) We assume that one of the following conditions hold:

i) $0<\alpha_{i}<a_{i+1}\left(s(t), u(t), x^{1}(t), . ., x^{i}(t)\right)<\beta_{i}$

ii) $0>-\alpha_{i}>a_{i+1}\left(s(t), u(t), x^{1}(t), . ., x^{i}(t)\right)>-\beta_{i}$

for every $t \geq 0$; for very $u \in \mathcal{U}$ and $1 \leq i \leq q$, where $\alpha_{i}$ and $\beta_{i}$ are constants.

Using hypothesis $H_{4}$ ), we can always assume that the nonlinear term $\gamma$ is global Lipschitz w.r.t. $x$. Indeed, if this is not the case, we can prolong $\gamma$ 
outside $\mathcal{K}^{\prime}$ to a global Lipschitz function (see for instance the construction given in section 4 formula (41)).

As above consider the symmetric positive definite matrix $S_{\theta}^{i}$ satisfying $\theta S_{\theta}^{i}+$ $A_{i}^{\top} S_{\theta}^{i}+S_{\theta}^{i} A_{i}=C_{i}^{\top} C_{i}$, and set

$$
\begin{aligned}
A & =\left(\begin{array}{cccc}
A_{1} & 0 & \ldots & 0 \\
0 & \ddots & \ddots & \vdots \\
\vdots & 0 & \ddots & 0 \\
0 & \ldots & 0 & A_{p}
\end{array}\right) \\
S_{\theta} & =\left(\begin{array}{cccc}
S_{\theta}^{1} & 0 & \ldots & 0 \\
0 & \ddots & \ddots & \vdots \\
\vdots & 0 & \ddots & 0 \\
0 & \ldots & 0 & S_{\theta}^{p}
\end{array}\right)
\end{aligned}
$$

As stated in remark 5 -a), $H_{5}$ ) can be reduced to $H_{5}-i$ ).

Now we can state our main result:

Theorem 7 Under hypotheses $\left.\left.H_{4}\right), H_{5}-i\right)$, the following system:

$$
\dot{\hat{x}}=\Omega(s, u, \breve{x}) A \hat{x}+\gamma(s, u, \breve{x})-\Lambda S_{\theta}^{-1} C^{\top}(C \hat{x}-y)
$$

is an exponential observer, for $\theta$ sufficiently large, where,

$$
\begin{aligned}
& \breve{x}=\left[\begin{array}{c}
\breve{x}^{1} \\
\cdot \\
\cdot \\
\cdot \\
\breve{x}^{p}
\end{array}\right] \text { and } \breve{x}^{i}=\left[\begin{array}{c}
y_{i} \\
\hat{x}_{2}^{i} \\
\cdot \\
\cdot \\
\hat{x}_{n_{i}}^{i}
\end{array}\right] \\
& \Lambda=\left[\begin{array}{cccc}
\lambda_{1} I_{1} & 0 & \cdots & 0 \\
0 & \ddots & \ddots & \vdots \\
\vdots & \ddots & \ddots & 0 \\
0 & \cdots & 0 & \lambda_{p} I_{p}
\end{array}\right] \\
& \Omega(s, u, \breve{x})=\left[\begin{array}{cccc}
a_{1}(s, u) I_{1} & 0 & \ldots & 0 \\
0 & a_{1}\left(s, u, \breve{x}^{1}\right) & \ddots & \vdots \\
\vdots & \ddots & \ddots & 0 \\
0 & \ldots & 0 & a_{p}\left(s, u, \breve{x}^{1}, . ., \breve{x}^{p-1}\right) I_{p}
\end{array}\right]
\end{aligned}
$$

$I_{i}$ is the $n_{i} \times n_{i}$ identity matrix, and $\lambda_{i} \geq \frac{\beta_{i}}{2}$. 
Proof. For the sake of simplicity, we only give the proof for $p=2$.

As above, set $e^{i}(t)=\hat{x}^{i}(t)-x^{i}(t), \bar{x}^{i}=\Delta_{\theta}^{i} x^{i}, \varepsilon^{i}=\Delta_{\theta}^{i} e^{i}$, where $\Delta_{\theta}^{i}$ is the $n_{i} \times n_{i}$ matrix $\operatorname{diag}\left(\frac{1}{\theta}, . ., \frac{1}{\theta^{n_{i}}}\right)$ we get:

$$
\begin{aligned}
\dot{\varepsilon}^{1}= & \theta\left[a_{1}(s, u) A_{1}-\lambda_{1}\left(S_{1}^{1}\right)^{-1} C_{1}^{T} C_{1}\right] \varepsilon^{1}+\Delta_{\theta} \tilde{\gamma}^{1} \\
\dot{\varepsilon}^{2}= & \theta\left[a_{2}\left(s, u, \breve{x}^{1}(t)\right) A_{2}-\lambda_{2}\left(S_{2}^{2}\right)^{-1} C_{2}^{T} C_{2}\right] \varepsilon^{2}+\Delta_{\theta} \tilde{\gamma}^{2} \\
& +\theta\left[a_{2}\left(s, u, \breve{x}^{1}\right)-\theta a_{2}\left(s, u, x^{1}(t)\right)\right] A_{2} \bar{x}^{2}
\end{aligned}
$$

where $\tilde{\gamma}^{i}=\gamma^{i}(s, u, \breve{x})-\gamma^{i}(s, u, x)$ and $S_{1}^{i}=S_{\theta}^{i}$ for $\theta=1$.

Now set $V_{i}=\left(\varepsilon^{i}\right)^{T} S_{1}^{i} \varepsilon^{i}$, and choose $\lambda_{1} \geq \frac{\beta_{1}}{2}$, as in the single output case (see formula (20)), we obtain:

$$
\dot{V}_{1} \leq-\left(\theta \alpha_{1}-2 \frac{\varkappa_{1}}{\lambda_{\min }^{1}}\right) V_{1}
$$

where $\varkappa_{1}$ is a constant which can be obtained in a similar way as in formula (19) by replacing $\tilde{\gamma}$ and $S$ by $\tilde{\gamma}^{1}$ and $S^{1}$ and $\lambda_{\text {min }}^{1}$ stands from the smallest eigenvalue of $S^{1}$.

Now taking $\theta>2 \frac{\varkappa_{1}}{\lambda_{\min }^{1}}$, it follows that:

$$
\left\|e^{1}(t)\right\| \leq c_{1}(\theta) \exp \left[-\frac{\left(\theta \alpha-2 \frac{\varkappa_{1}}{\lambda_{\min }^{1}}\right) t}{2}\right]
$$

for some constant $c_{1}(\theta)>0$.

Similarly, we have:

$$
\begin{aligned}
\dot{V}_{2}= & -\theta a_{2}\left(s, u, \breve{x}^{1}\right) V_{2}-\theta\left(2 \lambda_{2}-a\left(s, u, \breve{x}^{1}\right)\left(C_{2} \varepsilon^{2}\right)^{2}+2\left(\varepsilon^{2}\right)^{T} S_{1}^{2} \Delta_{\theta} \tilde{\gamma}^{2}\right. \\
& -2 \theta\left[a_{2}\left(s, u, \breve{x}^{1}\right)-a_{2}\left(s, u, x^{1}\right)\right]\left(\varepsilon^{2}\right)^{T} S_{1}^{2} A_{2} \bar{x}^{2}
\end{aligned}
$$

Using hypothesis $H_{5}-i$ ) and formula (23), it follows that there exists $t_{0}>0$, such that for every $t \geq t_{0}$,

$$
\alpha_{2}<a_{2}\left(s(t), u(t), \breve{x}^{1}(t)\right)<\beta_{2}
$$

As for (20), take $\lambda_{2} \geq \frac{\beta_{2}}{2}$, the following inequality hold for every $t \geq t_{0}$ :

$$
\dot{V}_{2} \leq-\left(\theta \alpha_{2}-2 \frac{\varkappa_{2}}{\lambda_{\min }^{2}}\right) V_{2}-2 \theta\left[a_{2}\left(s, u, \breve{x}^{1}\right)-a_{2}\left(s, u, x^{1}\right)\right]\left(\varepsilon^{2}\right)^{T} S_{1}^{2} A_{2} \bar{x}^{2}
$$

where $\varkappa_{2}$ is obtained as in formula (19) in which $\tilde{\gamma}$ and $S_{1}$ are replaced by $\tilde{\gamma}^{2}$ and $S_{1}^{2}$ and $\lambda_{\min }^{2}$ stands from the smallest eigenvalue of $S_{1}^{2}$.

Combining formula (23) and the fact that $x^{1}(t)$ is bounded $\left.\left(H_{4}\right)\right)$ and $a_{2}\left(s, u, x^{1}\right)$ is smooth w.r.t. $x^{1}$, we obtain:

$$
\left\|a_{2}\left(s(t), u(t), \breve{x}^{1}(t)\right)-a_{2}\left(s(t), u(t), x^{1}(t)\right)\right\| \leq \sigma(\theta) \exp \left[-\frac{\left(\theta \alpha-2 \frac{\varkappa_{1}}{\lambda_{\min }^{1}}\right) t}{2}\right]
$$

for some positive constant $\sigma(\theta)$.

To end the proof, it suffices to take $\theta>2 \max \left\{\frac{\varkappa_{1}}{\lambda_{\min }^{1}}, \frac{\varkappa_{2}}{\lambda_{\min }^{2}}\right\}$. 


\section{Failure diagnosis in a hydraulic process}

\subsection{Process description and modelling}

The hydraulic process consists of three cylindrical water tanks T1, T2, T3 (see Figure 1). The tank T1 contains cold water, and the water in the tank T2 is heated by means of a resistance. The water from tanks T1 and T2 are mixed in T3. Pneumatic valves V1, V2, V3, V4 and V5 are used as local regulators of the water flow rates through the system.

The model of the process can be obtained by combining the material and energy balances:

\section{Material Balance:}

This material balance is a classical one and uses only the relation between flow rates, levels of water and difference of pressure between the inlet and the outlet of valves.

We use the following notations:

$x_{i}$ : the level of water in tank $T_{i}, 1 \leq i \leq 3$

$u_{i}$ : the aperture of valve $i, 1 \leq i \leq 5$

$\alpha_{i}$ : the flow rate coefficient of valve $\mathrm{Vi}$

$P$ : the measured relative pressure at the inlet of valve $\mathrm{V} 1$ and $\mathrm{V} 2$.

$\rho$ : the water density

$g$ : the acceleration due to gravity

$l$ :the length of water column between the outlet of valves V3 and V4 and the inlet of tank T3 ( see Figure 1). The cross sectional area of cylindrical tanks $\mathrm{T} 1, \mathrm{~T} 2, \mathrm{~T} 3$ are $s_{1}, s_{2}$ and $s_{3}$ respectively.

We obtain:

$$
\begin{aligned}
& \dot{x}_{1}=\frac{\alpha_{1} \sqrt{P}}{s_{1}} u_{1}-\alpha_{3} \sqrt{\rho g} \frac{\sqrt{x_{1}+l}}{s_{1}} u_{3} \\
& \dot{x}_{2}=\frac{\alpha_{2} \sqrt{P}}{s_{2}} u_{2}-\alpha_{4} \sqrt{\rho g} \frac{\sqrt{x_{2}+l}}{s_{2}} u_{4} \\
& \dot{x}_{3}=\alpha_{3} \sqrt{\rho g} \frac{\sqrt{x_{1}+l}}{s_{3}} u_{3}+\alpha_{4} \sqrt{\rho g} \frac{\sqrt{x_{2}+l}}{s_{3}} u_{4}-\alpha_{5} \sqrt{\rho g} \frac{\sqrt{x_{3}}}{s_{3}} u_{5}
\end{aligned}
$$

\section{Energy balance:}

For a given tank Ti, we consider two global heat exchange coefficients: the first one, $h_{i}$, corresponds to the heat exchange coefficient between the free surface of the liquid in a tank (of area $s_{i}=\pi r_{i}^{2}$ ) and the surrounding environment, $r_{i}$ being the radius of the cylinder. The second one $h_{i}^{\prime}$ corresponds to heat exchange coefficient through the surface $S_{i}\left(S_{i}=\pi r_{i}^{2}+2 \pi r_{i} x_{i}\right)$ of the jacket of tank Ti. 
The experimental results showed that the heat exchange between the free surface T1 and the environment can be neglected. Thus we only consider the energy balances of tank T2 and T3.

Denote by $x_{4}$ and $x_{5}$ the temperatures of water in T2 and T3 which are assumed to be stirred. $\tau$ and $\tau^{\prime}$ denote the respective temperatures of water in $\mathrm{T} 1$ and the ambient which are assumed to be constant. Now set $u_{6}$ the power of the heating resistance in $\mathrm{T} 2$ and denote by $C_{p}$ the water heat capacity, the energy balance can be expressed as follows:

$$
\begin{aligned}
\dot{x}_{4}= & u_{2} \frac{\alpha_{2} \sqrt{P}}{x_{2} s_{2}}\left(\tau-x_{4}\right)+\frac{u_{6}}{\rho C_{p} s_{2} x_{2}}+\left[\frac{h_{2}}{\rho C_{P} x_{2}}+h_{2}^{\prime} \frac{2 \pi r_{2} x_{2}+s_{2}}{\rho s_{2} C_{P} x_{2}}\right]\left(\tau^{\prime}-x_{4}\right) \\
= & u_{2} \frac{\alpha_{2} \sqrt{P}}{x_{2} s_{2}}\left(\tau-x_{4}\right)+\frac{u_{6}}{\rho C_{p} s_{2} x_{2}}+\left[\frac{h_{2}+h_{2}^{\prime}}{\rho C_{P} x_{2}}+\frac{2 h_{2}^{\prime}}{\rho C_{P} r_{2}}\right]\left(\tau^{\prime}-x_{4}\right) \\
\dot{x}_{5}= & \frac{u_{3} \alpha_{3}}{x_{3} s_{3}} \sqrt{\rho g} \sqrt{x_{1}+l}\left(\tau-x_{5}\right)+\frac{u_{4} \alpha_{4}}{x_{3} s_{3}} \sqrt{\rho g} \sqrt{x_{2}+l}\left(x_{4}-x_{5}\right) \\
& +\left[\frac{h 3}{\rho C_{P} x_{3}}+h^{\prime} 3 \frac{2 \pi r_{3} x_{3}+s_{3}}{\rho s_{3} C_{P} x_{3}}\right]\left(\tau^{\prime}-x_{5}\right) \\
= & \frac{u_{3} \alpha_{3}}{x_{3} s_{3}} \sqrt{\rho g} \sqrt{x_{1}+l}\left(\tau-x_{5}\right)+\frac{u_{4} \alpha_{4}}{x_{3} s_{3}} \sqrt{\rho g} \sqrt{x_{2}+l}\left(x_{4}-x_{5}\right) \\
+ & {\left[\frac{h_{3}+h_{3}^{\prime}}{\rho C_{P} x_{3}}+\frac{2 h_{3}^{\prime}}{\rho C_{P} r_{3}}\right]\left(\tau^{\prime}-x_{5}\right) }
\end{aligned}
$$

To illustrate the methodology of section 2, we only consider the three following failure modes:

- The failure mode $v_{1}$ (resp. $v_{2}$ ) on the valve V3 (resp. V4) can be modelled as an additional unknown signal on the well-known control $u_{3}$ (resp. $u_{4}$ ).

- $v_{3}$ is the failure mode on the heating resistance. This failure can be considered as an additional unknown signal on $u_{6}$.

To achieve our objective, we take three output measurements at our disposal: $y_{1}=x_{2}$, the level of water in T2, $y_{2}=x_{3}$ that in T3, and $y_{3}=x_{5}$ is the water temperature in $\mathrm{T} 3$.

The constraints on the inputs $u_{i}$ of the process are:

$$
0.33 \leq u_{i} \leq 1, \quad i=1, \ldots, 5 ; 0 \leq u_{6} \leq 4.28
$$

The experimental physical domain $V$ of state variables that we considered is given by the following constraints:

$$
\left\{\begin{array}{c}
0<x_{1}<0.5 ; 0.3<x_{2}<0.5 ; 0.1<x_{3}<0.5 \\
\max \left(\tau, \tau^{\prime}\right)<x_{4}<80 ; \max \left(\tau, \tau^{\prime}\right)<x_{5}<80
\end{array}\right.
$$




\subsection{Detection and Isolation of $v_{1}$}

Noticing that the failure mode $v_{1}$ and $v_{2}$ occur in the material balance:

$$
\begin{aligned}
\dot{x}_{1}= & \frac{\alpha_{1} \sqrt{P}}{s_{1}} u_{1}-\alpha_{3} \sqrt{\rho g} \frac{\sqrt{x_{1}+l}}{s_{1}} u_{3}-\alpha_{3} \sqrt{\rho g} \frac{\sqrt{x_{1}+l}}{s_{1}} v_{1} \\
\dot{x}_{2}= & \frac{\alpha_{2} \sqrt{P}}{s_{2}} u_{2}-\alpha_{4} \sqrt{\rho g} \frac{\sqrt{x_{2}+l}}{s_{2}} u_{4}-\alpha_{4} \sqrt{\rho g} \frac{\sqrt{x_{2}+l}}{s_{2}} v_{2} \\
\dot{x}_{3}= & \alpha_{3} \sqrt{\rho g} \frac{\sqrt{x_{1}+l}}{s_{3}} u_{3}+\alpha_{4} \sqrt{\rho g} \frac{\sqrt{x_{2}+l}}{s_{3}} u_{4}-\alpha_{5} \sqrt{\rho g} \frac{\sqrt{x_{3}}}{s_{3}} u_{5} \\
& +\alpha_{3} \sqrt{\rho g} \frac{\sqrt{x_{1}+l}}{s_{1}} v_{1}+\alpha_{4} \sqrt{\rho g} \frac{\sqrt{x_{2}+l}}{s_{3}} v_{2}
\end{aligned}
$$

The output measurements are $y_{1}=x_{2}$ and $y_{2}=x_{3}\left(y=h(x)=\left(x_{2}, x_{3}\right)\right)$.

In order to achieve the FDI w.r.t. $v_{1}$, we will apply theorem 4 to system (30), (31), (32) with output $y_{1}, y_{2}$ :

Set $f=0, g_{1}=\frac{\alpha_{1} \sqrt{P}}{s_{1}} \frac{\partial}{\partial x_{1}}, g_{2}=\frac{\alpha_{2} \sqrt{P}}{s_{2}} \frac{\partial}{\partial x_{2}}, g_{3}=-\alpha_{3} \sqrt{\rho g} \frac{\sqrt{x_{1}+l}}{s_{1}} \frac{\partial}{\partial x_{1}}+$ $\alpha_{3} \sqrt{\rho g} \frac{\sqrt{x_{1}+l}}{s_{3}} \frac{\partial}{\partial x_{3}}, g_{4}=-\alpha_{4} \sqrt{\rho g} \frac{\sqrt{x_{2}+l}}{s_{2}} \frac{\partial}{\partial x_{2}}+\alpha_{4} \sqrt{\rho g} \frac{\sqrt{x_{2}+l}}{s_{3}} \frac{\partial}{\partial x_{3}}, g_{5}=0, \tilde{f}=0$, $\tilde{g}_{1}=\tilde{g}_{2}=\tilde{g}_{3}=\tilde{g}_{4}=0, \tilde{g}_{5}=-\alpha_{5} \sqrt{\rho g} \frac{\sqrt{x_{3}}}{s_{3}} \frac{\partial}{\partial x_{3}}, e_{1}=g_{3}$ and $e_{2}=g_{4}$. Under the above notations, system (30), (31), (32) takes the form (11), where the domain of state variables is $V=\left\{\left(x_{1}, x_{2}, x_{3}\right)^{T} ; 0<x_{1}<0.5,0.3<x_{2}<0.5,0.1<\right.$ $\left.x_{3}<0.5\right\}$ and the output $\left(y_{1}, y_{2}\right)=h(x)=\left(x_{2}, x_{3}\right)$.

In order to apply theorem 4 , let us calculate $\Delta^{*}$ and ker $d O_{\varphi \circ h}$.

Calculation of $\Delta^{*}$ :

$$
\Delta_{1}=\operatorname{span}\left\{e_{2}\right\}=\operatorname{span}\left\{-\frac{1}{s_{2}} \frac{\partial}{\partial x_{2}}+\frac{1}{s_{3}} \frac{\partial}{\partial x_{3}}\right\}
$$

$\left[f, e_{2}\right]=\left[g_{1}, e_{2}\right]=\left[g_{2}, e_{2}\right]=\left[g_{3}, e_{2}\right]=\left[g_{4}, e_{2}\right]=\left[g_{5}, e_{2}\right]=0$. Thus, $\Delta^{*}=$

$\Delta_{1}=\operatorname{span}\left\{-\frac{1}{s_{2}} \frac{\partial}{\partial x_{2}}+\frac{1}{s_{3}} \frac{\partial}{\partial x_{3}}\right\}$.

Consequently, $\varphi$ is given by $L_{e_{2}}(\varphi \circ h)=0$. This leads to: $\varphi o h(x)=\psi\left(s_{2} x_{2}+\right.$ $\left.s_{3} x_{3}\right)$ and a particular solution is given by:

$$
\varphi \circ h(x)=s_{2} x_{2}+s_{3} x_{3}
$$

A simple computation shows that ker $d O_{\varphi \circ h}$ is spanned by $\left\{s_{2} d x_{2}+s_{3} d x_{3}, d x_{1}\right\}$;

Now, let us check conditions i) and ii) of theorem 4.

Condition i): since $L_{e_{1}}(\varphi \circ h)=L_{g_{3}}\left(s_{2} x_{2}+s_{3} x_{3}\right)=\alpha_{3} \sqrt{\rho g} \sqrt{x_{1}+l} \neq 0$, then $e_{1} \notin \operatorname{ker} d O_{\varphi \circ h}$.

To show condition ii), we transform system (30), (31), (32) with output $\left(y_{1}, y_{2}\right)=\left(x_{2}, x_{3}\right)$ into the form (13), (14), (15). To do so, we perform a similar change of coordinates given by formula (15): $\xi_{1}=\bar{y}=\varphi \circ h(x)=s_{2} x_{2}-s_{3} x_{3}$, $\xi_{2}=L_{g_{3}}(\varphi \circ h)(x)=\alpha_{3} \sqrt{\rho g} \sqrt{x_{1}+l}$ and $\xi_{3}=x_{2}$. Hence system (30), (31), (32) with output $\bar{y}$, takes the following form: 


$$
\begin{aligned}
\dot{\xi}^{1} & =u_{3} A \xi^{1}+\gamma\left(u, y, \xi^{1}\right)+v_{1} E_{1}^{1}\left(u, \xi^{1}\right) \\
\dot{\xi}^{2} & =\omega(u, \xi)+v_{2} E_{2}^{2}\left(u, \xi^{1}\right) \\
\bar{y} & =C \xi^{1}
\end{aligned}
$$

where $\xi^{1}=\left[\begin{array}{ll}\xi_{1} & \xi_{2}\end{array}\right]^{T}, \xi^{2}=\xi_{3}$ and

$$
\begin{aligned}
A & =\left[\begin{array}{ll}
0 & 1 \\
0 & 0
\end{array}\right] \\
C & =\left[\begin{array}{ll}
1 & 0
\end{array}\right] \\
\gamma\left(u, y, \xi^{1}\right) & =\left[\begin{array}{ll}
\alpha_{2} \sqrt{P} u_{2}-\alpha_{5} \sqrt{\rho g} \sqrt{y_{2}} u_{5} & \frac{\alpha_{1} \alpha_{3}^{2} \rho g \sqrt{P}}{2 s_{1}} \frac{1}{\xi_{2}} u_{1}-\frac{\alpha_{3}^{2} \rho g}{2 s_{1}} u_{3}
\end{array}\right]^{T} \\
\omega(u, \xi) & =\frac{\alpha_{2} \sqrt{P}}{s_{2}} u_{2}-\frac{\alpha_{4} \sqrt{\rho g}}{s_{2}} \sqrt{\xi_{3}+l} u_{4} \\
E_{1}^{1}\left(u, \xi^{1}\right) & =\left[\begin{array}{ll}
\xi_{2} & -\frac{\alpha_{3}^{2} \rho g}{2 s_{1}}
\end{array}\right]^{T} \\
E_{2}^{2}\left(u, \xi^{1}\right) & =-\frac{\alpha_{4} \sqrt{\rho g}}{s_{2}} \sqrt{\xi_{3}+l}
\end{aligned}
$$

To verify condition ii) of theorem 4 , it suffices to show that subsystem (33), (35) in which $v_{1}=0$ admits an asymptotic observer. To achieve this objective, we only need to show that system (33), (35) satisfies hypotheses H2), H3) stated in subsection 3.1

Hypothesis $\mathrm{H} 2$ ) is obviously satisfied.

To obtain H3), we extend $\gamma\left(u, y, \xi^{1}\right)$ to a global Lipschitz function $\tilde{\gamma}$ so that the obtained nonlinear term becomes a global Lipschitz function. According to the physical domain given by (29), $\tilde{\gamma}$ can be obtained by replacing $\frac{1}{\xi_{2}}$ by the function:

$$
\left\{\begin{array}{c}
\frac{1}{\xi_{2}} \quad \text { if } \xi_{2}=\alpha_{3} \frac{s_{2}}{s_{3}} \sqrt{\rho g} \sqrt{x_{1}+l} \geq \alpha_{3} \frac{s_{2}}{s_{3}} \sqrt{\rho g} \sqrt{l} \\
\sqrt{l} \text { if } \xi_{2} \leq \sqrt{l}
\end{array}\right.
$$

Consequently, the obtained nonlinear term becomes a global Lipschitz function. Finally, the residual generator takes the form:

$$
\left\{\begin{array}{l}
\dot{\zeta}=u_{3} A \zeta+\tilde{\gamma}(u, y, \zeta)-\rho S_{\theta}^{-1} C^{\top}(C \zeta-\bar{y}) \\
r=C \zeta-\bar{y}
\end{array}\right.
$$

$$
\text { where } \zeta=\left[\begin{array}{ll}
\zeta_{1} & \zeta_{2}
\end{array}\right]^{T}, S_{\theta}=\left[\begin{array}{cc}
\frac{1}{\theta} & -\frac{1}{\theta^{2}} \\
-\frac{1}{\theta^{2}} & \frac{2}{\theta^{3}}
\end{array}\right] \text {. }
$$

\subsection{Detection and Isolation of $v_{2}$}

The failure model that we will consider takes the form : 


$$
\dot{x}_{2}=\frac{\alpha_{2} \sqrt{p}}{s_{2}} u_{2}-u_{4} \alpha_{4} \sqrt{\rho g} \frac{\sqrt{x_{2}+l}}{s_{2}}+v_{2} \alpha_{4} \sqrt{\rho g} \frac{\sqrt{x_{2}+l}}{s_{2}}
$$

and the output measurement is $y_{2}=x_{2}$.

Obviously, the following system:

$$
\left\{\begin{array}{l}
\dot{\hat{x}}_{2}=\frac{\alpha_{2} \sqrt{p}}{s_{2}} u_{2}-u_{4} \alpha_{4} \sqrt{\rho g} \frac{\sqrt{y_{2}+l}}{s_{2}}-k\left(\hat{x}_{2}-x_{2}\right) \\
r_{2}=\hat{x}_{2}-y_{2}
\end{array}\right.
$$

is a residual filter which detects and isolates $v_{2}$, where $k>0$ is a constant.

\subsection{Detection of $v_{3}$}

In this case, we only consider the detection of $v_{3}$. More precisely, the residual filter that we will consider only detects $v_{3}$ in the absence of $v_{1}, v_{2}$.

The on-line measurements are $y_{1}=x_{2}, y_{2}=x_{3}$ and $y_{3}=x_{5}$. Using equations (24), (26), (27) and (28), we obtain the following reduced model:

$$
\left\{\begin{array}{l}
\dot{x}_{1}=\frac{\alpha_{1} \sqrt{P}}{s_{1}} u_{1}-\alpha_{3} \sqrt{\rho g} \frac{\sqrt{x_{1}+l}}{s_{1}} u_{3} \\
\dot{x}_{3}=\alpha_{3} \sqrt{\rho g} \frac{\sqrt{x_{1}+l}}{s_{3}} u_{3}+\alpha_{4} \sqrt{\rho g} \frac{\sqrt{y_{1}+l}}{s_{3}} u_{4}-\alpha_{5} \sqrt{\rho g} \frac{\sqrt{x_{3}}}{s_{3}} u_{5} \\
\dot{x}_{4}=\frac{\alpha_{2} \sqrt{P}}{y_{1} s_{2}}\left(\tau-x_{4}\right) u_{2}+\frac{u_{6}}{\rho C_{p} s_{2} y_{1}}+\left[\frac{h_{2}+h_{2}^{\prime}}{\rho C_{P} y_{1}}+\frac{2 h_{2}^{\prime}}{\rho C_{P} r_{2}}\right]\left(\tau^{\prime}-x_{4}\right) \\
\dot{x}_{5}=\frac{u_{3} \alpha_{3}}{x_{3} s_{3}} \sqrt{\rho g} \sqrt{x_{1}+l}\left(\tau-x_{5}\right)+\frac{u_{4} \alpha_{4}}{x_{3} s_{3}} \sqrt{\rho g} \sqrt{y_{1}+l}\left(x_{4}-x_{5}\right)
\end{array}\right.
$$

First, we show that such system admits an exponential observer. Next, we give a residual filter which detect the failure $v_{3}$ in the absence of $v_{1}$ and $v_{2}$.

To show that system (38) with output $\bar{y}=\left(y_{2}, y_{3}\right)=\left(x_{3}, x_{5}\right)$ admits an exponential observer, we transform this system into the cascade form given in subsection 3.2 and we apply theorem 7 .

To do so, consider the change of coordinates $z_{1}=x_{3}, z_{2}=\sqrt{x_{1}+l}, z_{3}=x_{5}$, $z_{4}=x_{4}$ and set $z^{1}=\left(\begin{array}{c}z_{1} \\ z_{2}\end{array}\right), z^{2}=\left(\begin{array}{c}z_{3} \\ z_{4}\end{array}\right)$, we obtain the following cascade system:

$$
\begin{aligned}
\dot{z}^{1} & =a_{1}(s, u) A_{1} z^{1}+\gamma^{1}\left(u, s, z^{1}\right) \\
\dot{z}^{2} & =a_{2}\left(s, u, z^{1}\right) A_{2} z^{2}+\gamma^{2}\left(u, s, z^{1}, z^{2}\right) \\
\bar{y} & =\left(\bar{y}_{1}, \bar{y}_{2}\right)=\left(z_{1}, z_{3}\right)
\end{aligned}
$$


where, $u=\left(u_{1}, \ldots, u_{6}\right), s=y_{1}=x_{2}$ the known measurement and,

$$
\begin{aligned}
& A_{1}=A_{2}=\left[\begin{array}{ll}
0 & 1 \\
0 & 0
\end{array}\right] \\
& a_{1}(s, u)=\frac{\alpha_{3} \sqrt{\rho g}}{s_{3}} u_{3} \\
& a_{2}\left(s, u, z^{1}\right)=\frac{u_{4} \alpha_{4}}{z_{1} s_{3}} \sqrt{\rho g} \sqrt{y_{1}+l} \\
& \gamma^{1}\left(u, s, z^{1}\right)=\left[\begin{array}{c}
\alpha_{4} \sqrt{\rho g} \frac{\sqrt{y_{1}+l}}{s_{3}} u_{4}-\alpha_{5} \sqrt{\rho g} \frac{\sqrt{z_{1}}}{s_{3}} u_{5} \\
\frac{1}{2 z_{2}} \frac{\alpha_{1} \sqrt{P}}{s_{1}} u_{1}-\frac{\alpha_{3}}{2 s_{1}} \sqrt{\rho g} u_{3}
\end{array}\right] \\
& \frac{u_{3} \alpha_{3}}{z_{1} s_{3}} \sqrt{\rho g} z_{2}\left(\tau-z_{3}\right)-\frac{u_{4} \alpha_{4}}{z_{1} s_{3}} \sqrt{\rho g} \sqrt{y_{1}+l} z_{3} \\
& \gamma^{2}\left(u, s, z^{1}, z^{2}\right)=\left[\begin{array}{c}
\alpha_{2} \sqrt{P} \\
\frac{\alpha_{2}}{y_{1} s_{2}}\left(\tau-z_{4}\right) u_{2}+\frac{u_{6}}{\rho C_{p} s_{2} y_{1}}+\left[\frac{h_{2}+h_{2}^{\prime}}{\rho C_{P} y_{1}}+\frac{2 h_{2}^{\prime}}{\rho C_{P} r_{2}}\right]\left(\tau^{\prime}-z_{4}\right)
\end{array}\right]
\end{aligned}
$$

Clearly, $H_{4}$ ) and $H_{5}$ ) (see subsection 3.2 are satisfied.

Using similar notations as in section 3 , the observer takes the form:

$$
\left\{\begin{array}{c}
\dot{\bar{z}}^{1}=a_{1}(s, u) A_{1} \hat{z}^{1}+\breve{\gamma}^{1}\left(u, s, \breve{z}^{1}\right)-\lambda_{1}\left(S_{\theta}^{1}\right)^{-1}\left(\hat{z}_{1}-\bar{y}_{1}\right) \\
\dot{\bar{z}}^{2}=a_{2}\left(s, u, \breve{z}^{1}\right) A_{2} \hat{z}^{2}+\gamma^{2}\left(u, s, \breve{z}^{1}, \breve{z}^{2}\right)-\lambda_{2}\left(S_{\theta}^{2}\right)^{-1}\left(\hat{z}_{3}-\bar{y}_{2}\right)
\end{array}\right.
$$

Here,

$$
\begin{aligned}
& \breve{z}^{i}=\left(\begin{array}{c}
\bar{y}_{i} \\
\hat{z}_{2}^{i}
\end{array}\right) \\
& \breve{\gamma}^{1}\left(u, s, \breve{z}^{1}\right)=\left[\begin{array}{c}
\alpha_{4} \sqrt{\rho g} \frac{\sqrt{y_{1}+l}}{s_{3}} u_{4}-\alpha_{5} \sqrt{\rho g} \frac{\sqrt{y_{1}}}{s_{3}} u_{5} \\
\frac{1}{2 \alpha\left(\hat{z}_{2}\right)} \frac{\alpha_{1} \sqrt{P}}{s_{1}} u_{1}-\frac{\alpha_{3}}{2 s_{1}} \sqrt{\rho g} u_{3}
\end{array}\right] \\
& \alpha\left(\hat{z}_{2}\right)=\left\{\begin{array}{c}
\hat{z}_{2} \text { if } \hat{z}_{2}>\sqrt{l} \\
\sqrt{l} \text { if } \hat{z}_{2}<\sqrt{l}
\end{array}\right. \\
& a_{2}\left(s, u, \breve{z}^{1}\right)=\frac{u_{4} \alpha_{4}}{\bar{y}_{1} s_{3}} \sqrt{\rho g} \sqrt{y_{1}+l} \\
& \breve{\gamma}^{2}\left(u, s, \breve{z}^{1}, \breve{z}^{2}\right)=\left[\begin{array}{c}
\frac{u_{3} \alpha_{3}}{\bar{y}_{1} s_{3}} \sqrt{\rho g} \hat{z}_{2}\left(\tau-\bar{y}_{2}\right)-\frac{u_{4} \alpha_{4}}{\bar{y}_{1} s_{3}} \sqrt{\rho g} \sqrt{y_{1}+l} \bar{y}_{2} \\
\frac{\alpha_{2} \sqrt{P}}{y_{1} s_{2}}\left(\tau-\hat{z}_{4}\right) u_{2}+\frac{u_{6}}{\rho C_{p} s_{2} y_{1}}+\left[\frac{h_{2}+h_{2}^{\prime}}{\rho C_{P} y_{1}}+\frac{2 h_{2}^{\prime}}{\rho C_{P} r_{2}}\right]\left(\tau^{\prime}-\hat{z}_{4}\right)
\end{array}\right]
\end{aligned}
$$

Note that if we initialize systems (39) and (40) at the same initial state belonging to the physical domain corresponding to the physical constraints (29), then the two trajectories $z(t), \hat{z}(t)$ coincide. Moreover, we have:

$$
\begin{aligned}
\gamma^{1}\left(u(t), s(t), z^{1}(t)\right) & =\breve{\gamma}^{1}\left(u(t), s(t), \breve{z}^{1}(t)\right) \\
\gamma^{2}\left(u(t), s(t), z^{1}(t), z^{2}(t)\right) & =\breve{\gamma}^{2}\left(u(t), s(t), \breve{z}^{1}(t), \breve{z}^{2}(t)\right) \\
\text { for every } t & \geq 0
\end{aligned}
$$


Since $\breve{\gamma}^{1}\left(u, s, \breve{z}^{1}\right)$ and $\breve{\gamma}^{2}\left(u, s, \breve{z}^{1}, \breve{z}^{2}\right)$ are global Lipschitz w.r.t. $\left(\hat{z}_{2}, \hat{z}_{4}\right)$, it follows that for an adequate choice of $\theta, \lambda_{1}$ and $\lambda_{2}$ (see theorem 9) system (40) becomes an exponential observer.

Finally, the residual filter of $v_{3}$ is given by (40) and the following system:

$$
r_{3}=\hat{z}_{3}-y_{3}
$$

\subsection{Experimental results}

To show the performance of the residual generators proposed above, we did the following experiment:

From $t=150 \mathrm{~s}$ until $t=200 \mathrm{~s}$ (resp. from $t=450 \mathrm{~s}$ until $t=500 \mathrm{~s}$ ), we varied the aperture of the valve $v_{3}$ (resp. $v_{4}$ ), see figure (3.a). The corresponding residual generators are given by the dynamical systems (36) and (37). The gain parameters are $\rho=10, \theta=0.002, k=0.04$. Clearly, figures (3.b) and (3.c) show that residuals $r_{1}$ and $r_{2}$ detect and isolate their corresponding failures.

From $t=900 \mathrm{~s}$ until $t=950 \mathrm{~s}$ we varied the power of the heating resistance (see figure 4.a), as it was shown in section 4.4 the residual given by (40) is sensitive to all faults $v_{1}, v_{2}$ and $v_{3}$. The parameters of filter (40) are $\lambda_{1}=$ $0.2, \lambda_{2}=0.1, \quad \theta=0.4$. In this case, the residual generator $r_{3}$ only detects $v_{3}$. However, a logical decision permits to isolate the fault $v_{3}$.

\section{Conclusion}

In this paper, we presented a nonlinear approach to solve the problem of failure detection and isolation. The proposed approach deals with a nonlinear extension of the linear geometric approach proposed by Massoumnia et al.,[27]. We have shown that our contribution corresponds to a sufficient condition and provides a design procedure to solve the problem. We illustrated the methodology through a hydraulic process. Three kinds of failures are considered and the design of residual generators is given, these residual generators allows us to detect and isolate two failure modes, the third one can be detected by the third residual generator and isolated by a logical decision.

The performance of the residual generators is shown and gives satisfying results.

\section{References}

[1] E. G. Alcorta and P. M. Frank. Deterministic nonlinear observer-based approaches to fault diagnosis: A survey. Control Eng. Practice, 5(5):663$670,1997$.

[2] M. Basseville. Detecting changes in signals and systems. Automatica, 24:309-326, 1998. 
[3] R. V. Beard. Fault accomodation in linear systems through selfreorganization. $\mathrm{PhD}$ thesis, Massachussetts institutes of technology, Cambridge, Massachussetts, U.S.A., 1971.

[4] G. Bornard and H. Hammouri. A high gain observer for a class of nonlinear systems under locally regular inputs. Proceedings of the 30th CDC IEEE, Brighton, G.B., 1991.

[5] G. Bornard, N.Couenne, and F. Celle. Regularly persistent observers for bilinear systems. Proceedings of the 29th international conference on non linear systems, New trends in non linear systems, 1988.

[6] F. Deza and J. P. Gauthier. Nonlinear observer for distillation columns. Proceedings of the 30th CDC IEEE, Brighton, G.B., 1991.

[7] P. M. Frank. Fault diagnosis in dynamic systems using analytical and knowledge-based redundancy - a survey and some new results. Automatica, 26(3):459-474, 1990.

[8] P. M. Frank and X. Ding. Survey of robust residual generation and evaluation methods in observer-based fault detection systems. J. of Process Control, 7:403-424, 1997.

[9] P. M. Frank and R. Seliger. Fault detection and isolation in automatic processes, control and dynamic systems. Academic Press, 49:241-287, 1991.

[10] J. P. Gauthier and G. Bornard. Observability for any $\mathrm{u}(\mathrm{t})$ of a class of nonlinear systems. IEEE Transaction on Automatic Control, 26(4):922926, 1981.

[11] J. P. Gauthier, H. Hammouri, and S. Othman. A simple observer for nonlinear systems - application to a bioreactor. IEEE Transaction on Automatic Control, 37(6):875-880, 1992.

[12] J. P. Gauthier and I. Kupka. Observability and observers for nonlinear systems. SIAM Journal of Control and Optimization, 32(4):975-994, 1994.

[13] J. Gertler. Fault detection and isolation using parity relations. Control Engineering Practice, (5):653-661, 1997.

[14] H. Hammouri and J. DeLeon. Topological properties of observer's inputs. Analysis of controlled dynamical systems, pages 231-242, 1990.

[15] H. Hammouri and J. P. Gauthier. Bilinearization up to output injection. Systems $\&$ Control Letters, (11):139-149, 1988.

[16] H. Hammouri and J. P. Gauthier. Global time varying linearization up to output injection. SIAM J. Control and Optimization, (6):1259-1310, 1992. 
[17] H. Hammouri and M. Kinnaert. A new procedure for time-varying linearization up to output injection. Systems \& Control Letters, 28:151-157, 1996.

[18] H. Hammouri, M. Kinnaert, and E. ElYaagoubi. Residual generator synthesis for bilinear systems up to output injection. proceedings of the 33rd CDC IEEE, 2:1548-1553, 1994.

[19] H. Hammouri, M. Kinnaert, and E. ElYaagoubi. Fault detection and isolation for state affine systems. European Journal of Control, 4(1), 1998.

[20] H. Hammouri, M. Kinnaert, and E. ElYaagoubi. Observer-based approach to fault detection and isolation for nonlinear systems. IEEE Transactions on automatic control, 44(10), 1999.

[21] R. Isermann. Supervision, fault-detection and fault-diagnosis methods - an introduction. Control Engineering Practice, 5(5):639-652, 1997.

[22] A. Isidori. Nonlinear Control Systems : an introduction. Springer Verlag, 3rd Edition, 1995.

[23] H. Jones. Failure detection in linear systems. PhD thesis, Massachussetts institutes of technology, Cambridge, Massachussetts, U.S.A., 1973.

[24] A. Krener and A. Isidori. Linearization by output injection and nonlinear observers. Systems $\&$ Control Letters, (3):47-52, 1983.

[25] A. Krener and W. Respondek. Nonlinear observers with linearizable error dynamics. SIAM J. Control and Optimization, 23:197-216, 1985.

[26] M. A. Massoumnia. A geometric approach to the synthesis of failure detection filters. IEEE Transaction on Automatic Control, 31(9):839-846, 1986 .

[27] M. A. Massoumnia, G. C. Verghese, and A. S. Willsky. Failure detection and identification. IEEE Transaction on Automatic Control, 34(3):316-321, 1989.

[28] H. Nijmeijer and A. Van der Schaft. Nonlinear Dynamical Control Systems. Springer Verlag, Berlin, 1990.

[29] R. J. Patton and J. Chen. Robust fault detection using eigenstructure assignment: A tutorial consideration and some new results. Control Engineering Practice, 5(5):671-682, 1997.

[30] C. De Persis and A. Isidori. On the observability codistributions of a nonlinear system. Systems \& Control Letters, (40):297-304, 2000.

[31] R. Seliger and P. M. Frank. Advances in fault diagnosis for dynamic systems: Model-based approaches. Nonlinear uncertain systems, R. Patton, P. M. Frank, R. N. Clark (eds.). Springer Verlag, 2000. 
[32] Xiao-Hua Xia and Wei-Bin Gao. Nonlinear observer design by observer error linearization. SIAM J. Control and Optimization, 27:199-216, 1989. 


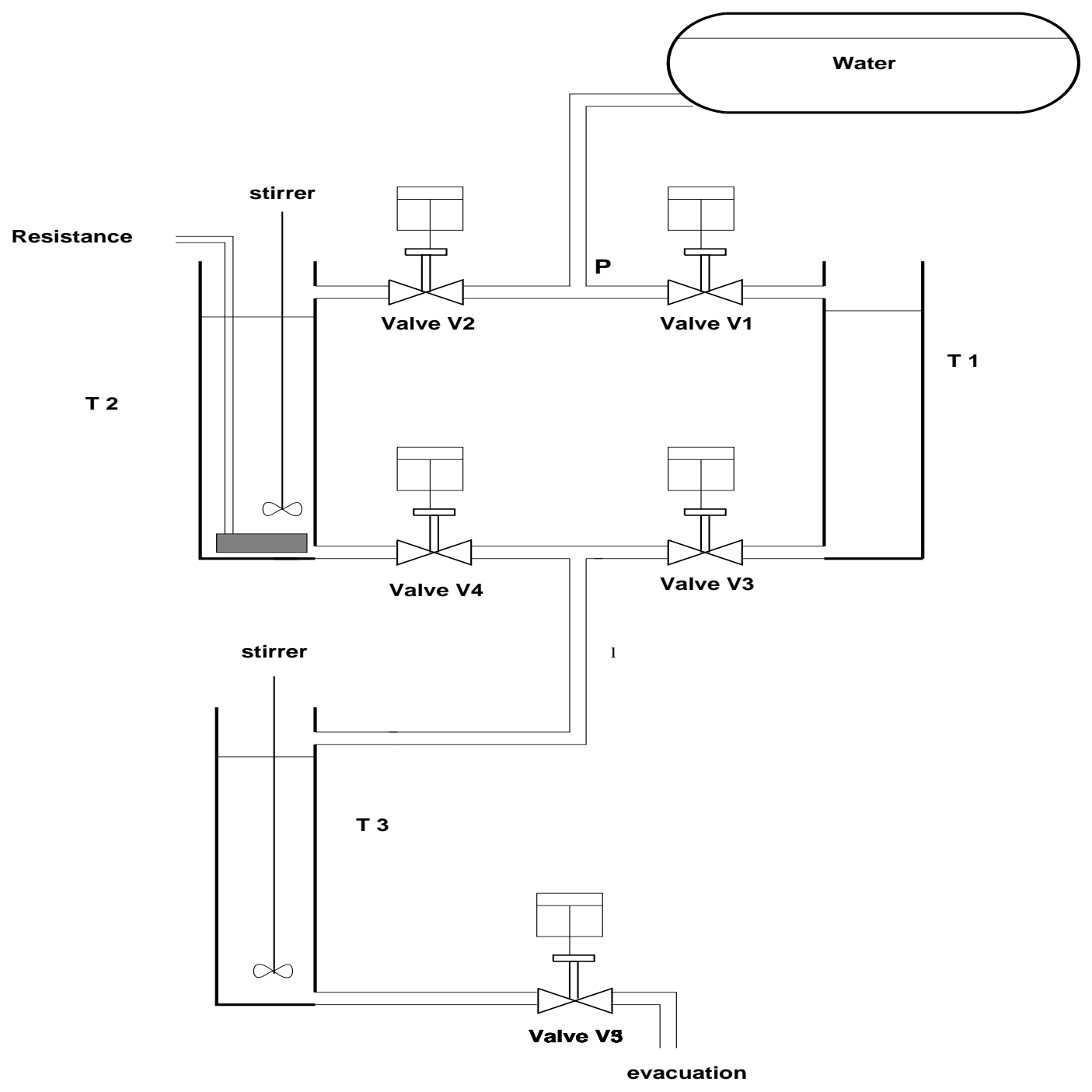

figure 1 


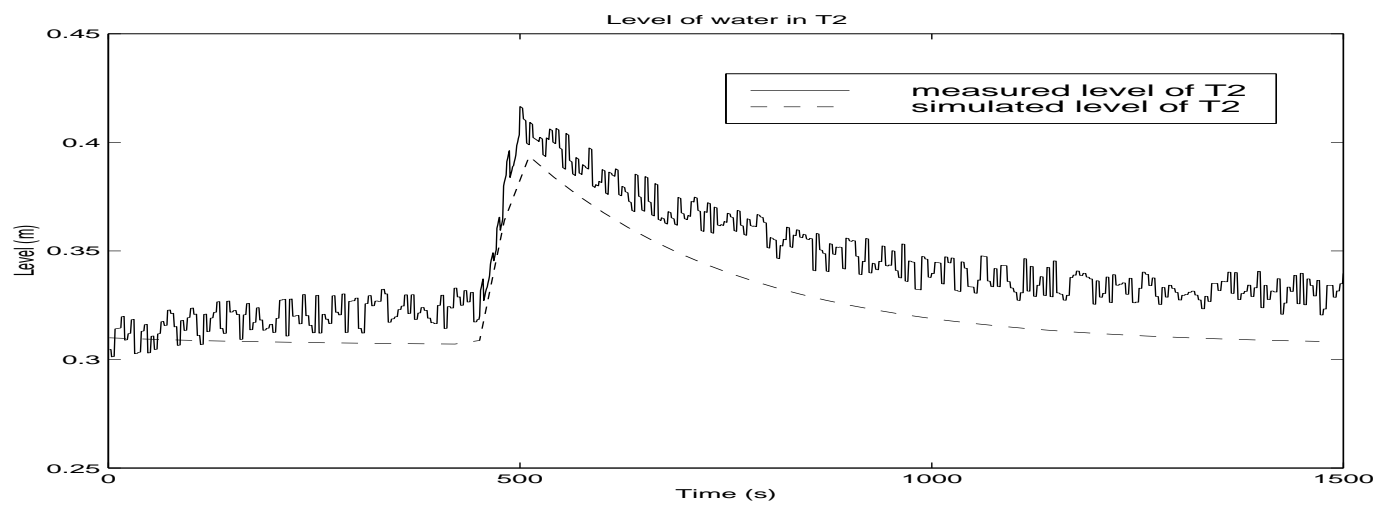

figure 2.a
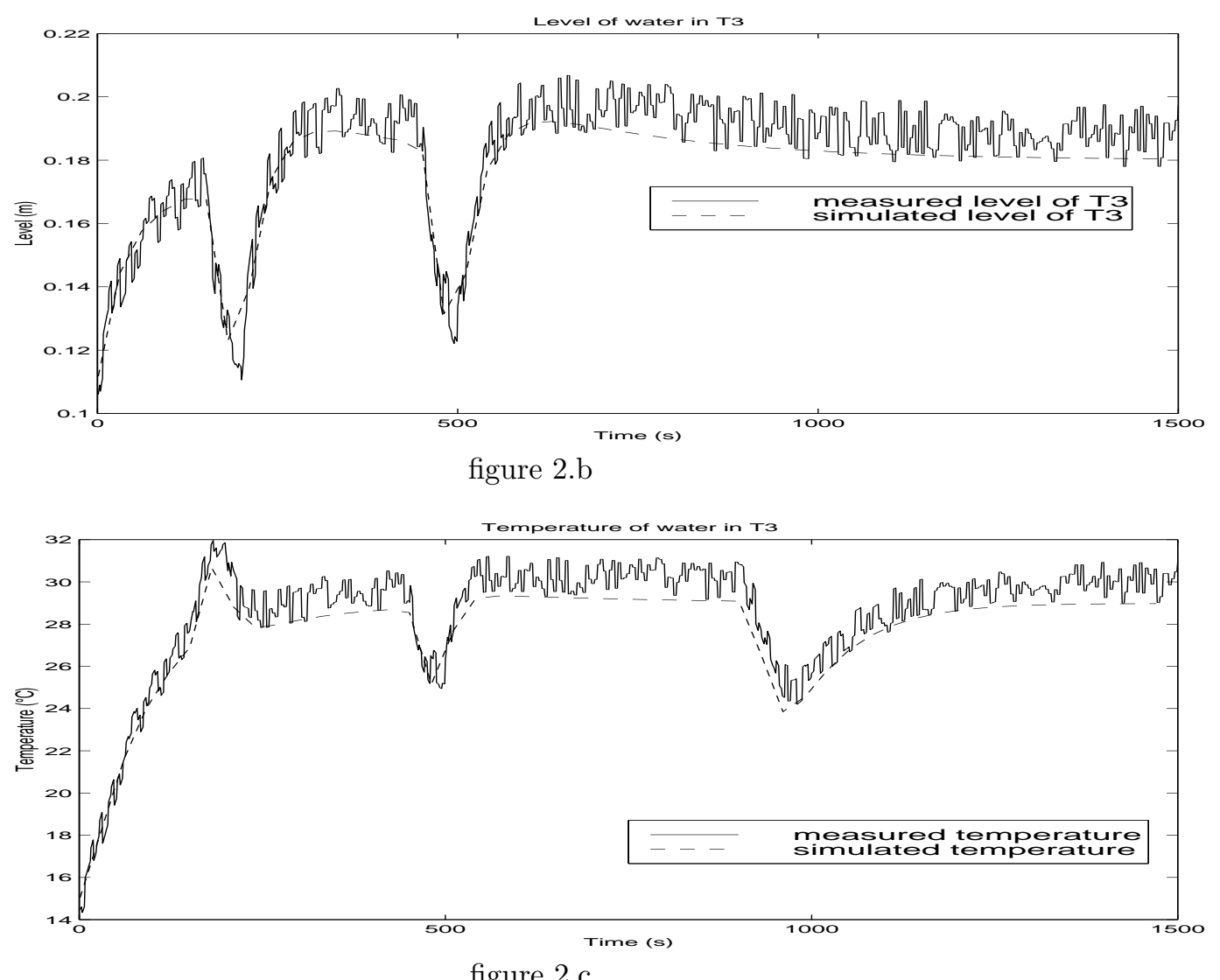


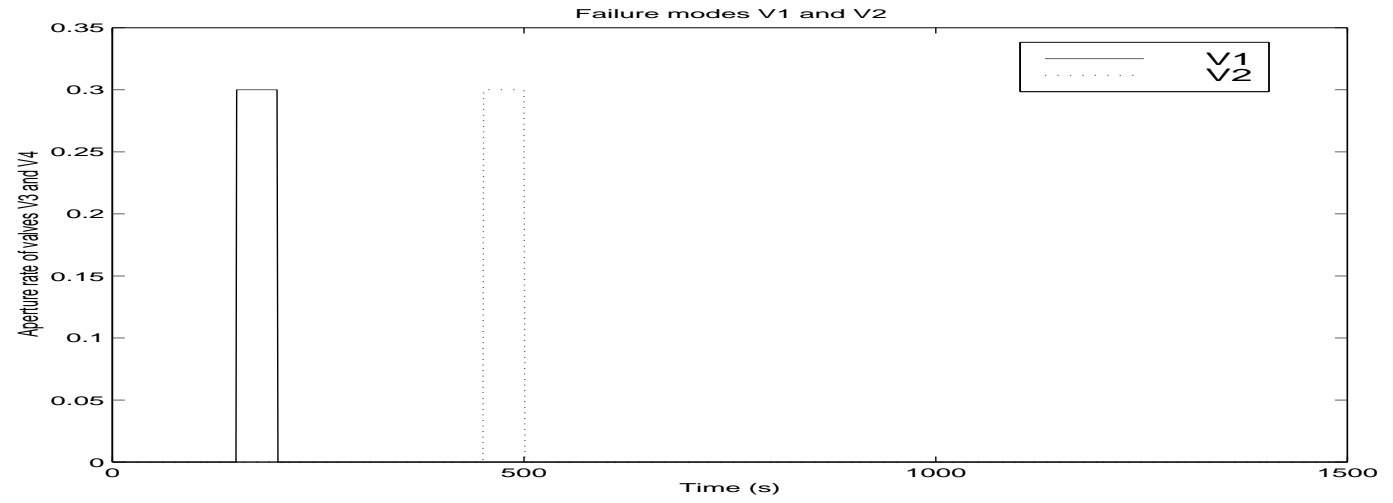

figure $3 . \mathrm{a}$

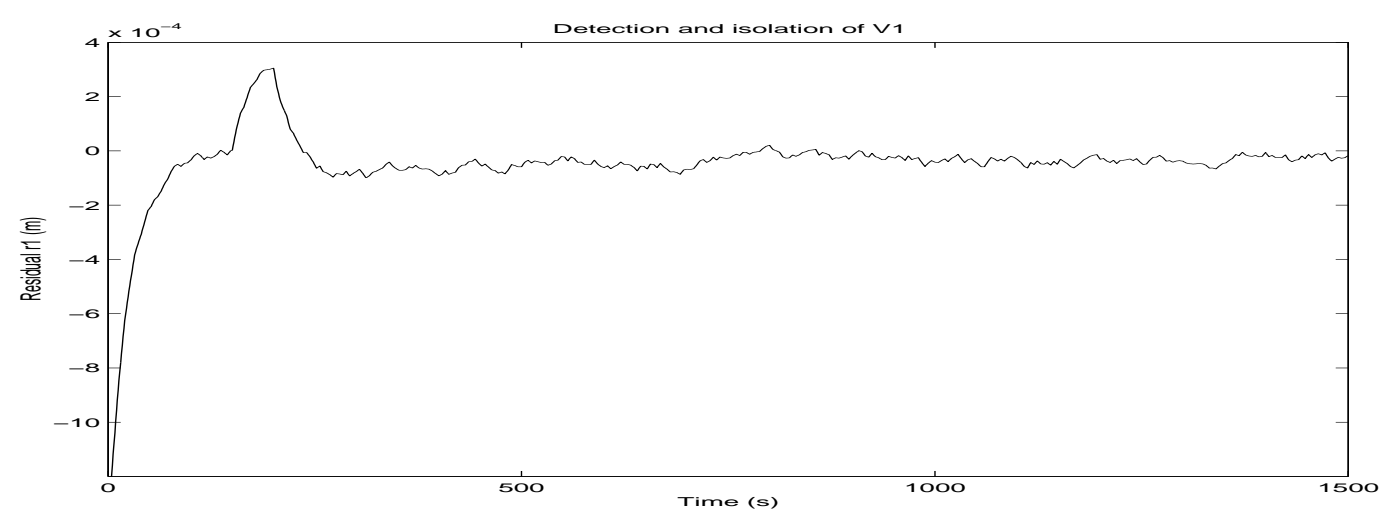

figure 3.b

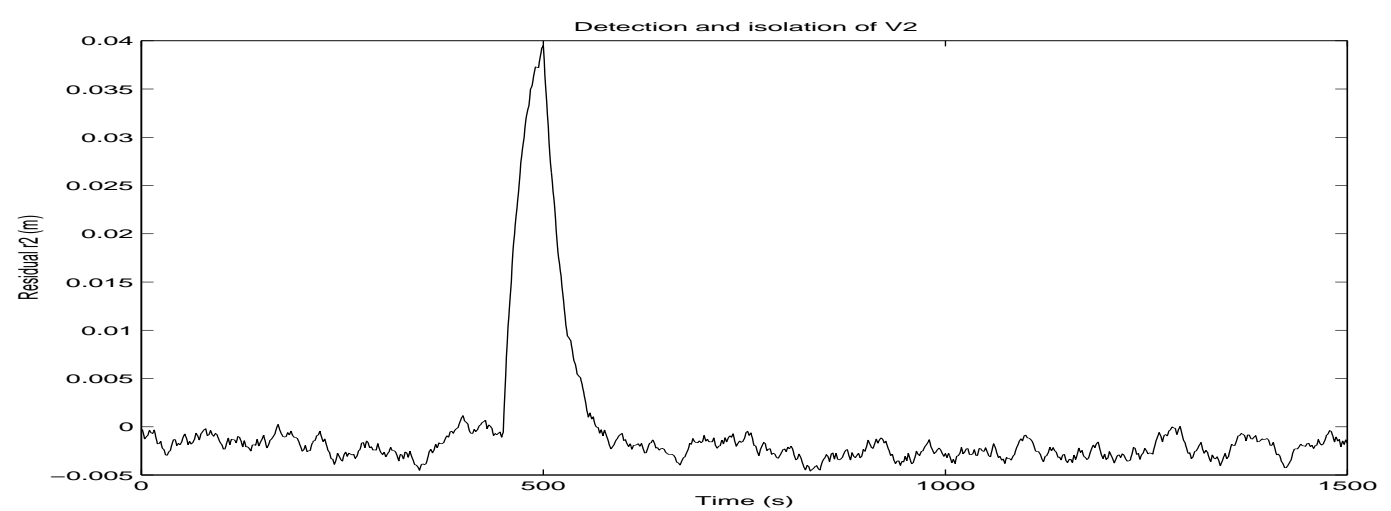

figure 3.c 


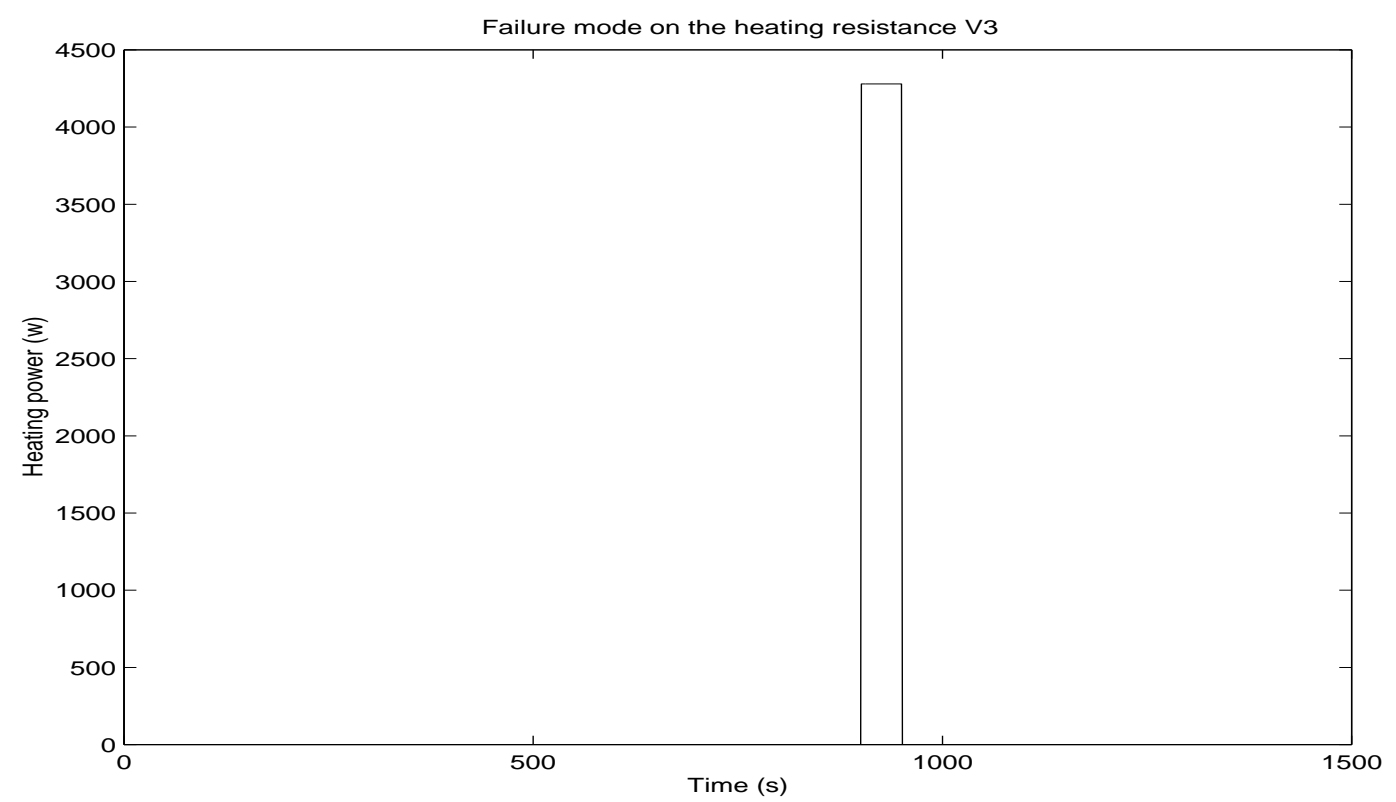

figure 4.a

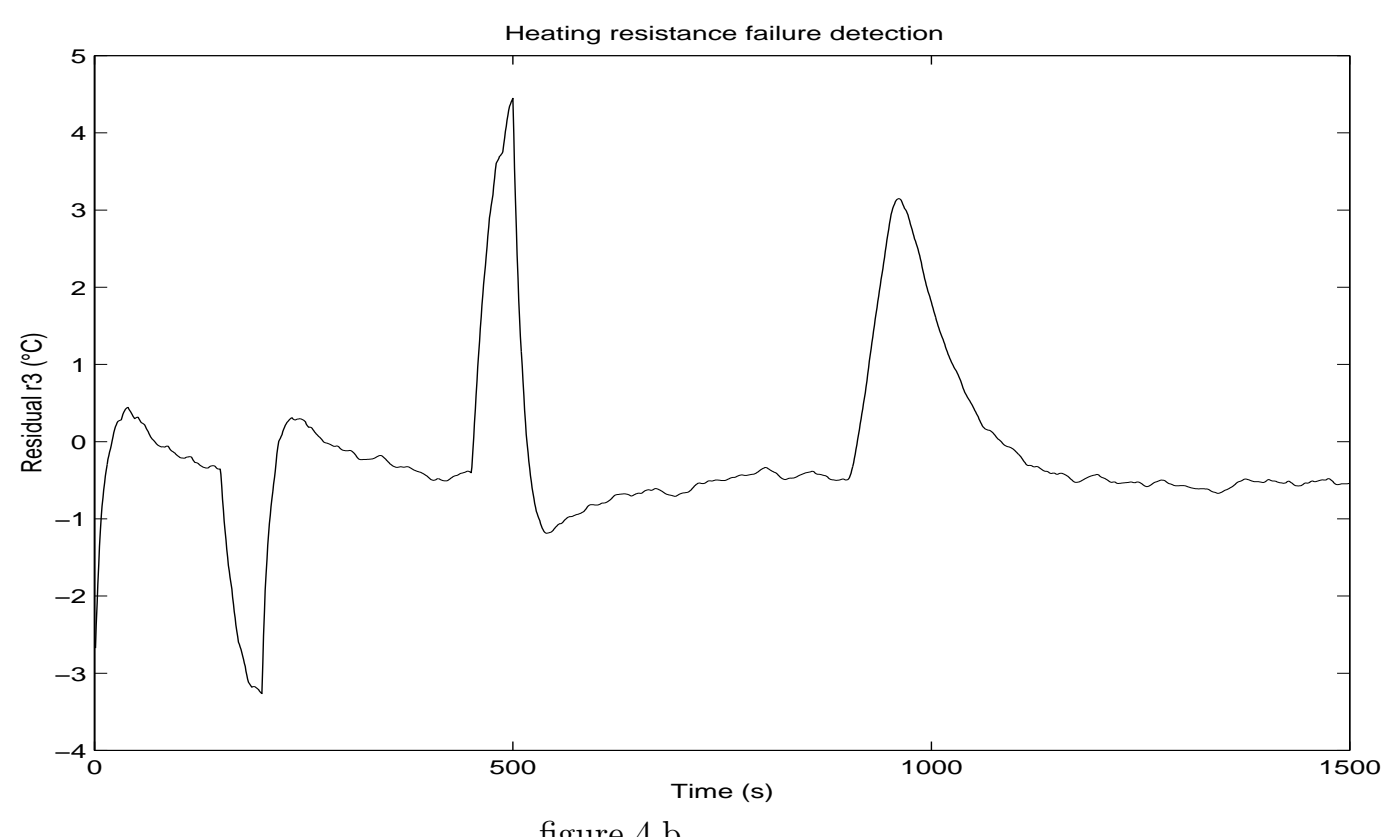

figure 4.b 